\title{
Content Based Image Retrieval Using 2-D Discrete Wavelet with Texture Feature with Different Classifiers
}

\author{
Mohankumar $\mathrm{C}^{1}$, Madhavan $\mathrm{J}^{2}$ \\ ${ }^{I}(P G$ Scholar in Communication Systems, Adhiyamaan College Of Engineering, Hosur, India) \\ ${ }_{2}^{2}$ (AP in Electronics And Communication Engineering, Adhiyamaan College Of Engineering, Hosur, India)
}

\begin{abstract}
In this paper we proposed discrete wavelet transform with texture for content based image retrieval. Our proposed method uses 2-D Discrete Wavelet Transform for reducing the Dimensions of test image and trained images. Further gray level co-occurrence matrix is applied for all test and trained images of LL components of level 2 decomposed images for extract the texture feature of the images. Then similar images are retrieved by using different distance classifiers. Experimental results are performed for Wang's database and it gives the improved performance for homogeneity with energy property of GLCM of texture feature for Euclidean distance method.
\end{abstract}

Keywords-CBIR, Texture, 2-D Discrete Wavelet Transform, Euclidean distance, Manhattan distance.

\section{Introduction}

Content Based Image Retrieval is to retrieve an image from the image database when given a query image. Query Image is the users target image for the searching process. CBIR systems operate in two phases: indexing and searching. In the indexing phase, each image of the database is represented using a set of image attribute, such as color, shape, texture and layout. Extracted features are stored in a feature database. In the searching phase, when a user makes a query, a feature vector for the query is computed. Using a similarity criterion, this vector is compared to the vectors in the feature database.

The images most similar to the query are returned to the user. Rapid advances in hardware technology and growth of computer power make facilities for spread use of World Wide Web. This causes that digital libraries manipulate huge amounts of image data. Due to the limitations of space and time, the images are represented in compressed formats. Therefore, new waves of research efforts are directed to feature extraction in compressed domain. Wavelet transform can be used to characterize textures using statistical properties of gray levels of the pixels comprising a surface image [4]. The wavelet transform is a tool that cuts up data or functions or operations into different frequency components and then studies each component with a resolution matched to its scale. In the proposed paper, we use 2-D Discrete Wavelet transform with textured feature images. We also provide a retrieval accuracy strategy for different wavelets.

The outline of the paper is as follows. General structure of proposed system is reviewed in section 2. In section 3 discrete wavelet transform is described. Section 4 and 5 discussed about Texture and distance measures. Experimental results and conclusions are presented in section 6 and 7 respectively.

\section{General Structure Of Proposed CBIR System}

Fig.1 shows the basic block diagram of Content based image retrieval system. Our Proposed method compares the performance of Content based image retrieval using DWT with Texture for similarity matching of Euclidian distance(L2), Manhattan distance(L1) and Standard Euclidean distance(std L2) method.

All train images decomposed using discrete wavelet transform. After DWT, we are taking only low frequency components (LL) of the image for texture feature using GLCM. Then the final feature vectors of all the train images are stored in the database. Same process is done for query image. Finally first 5 similar images are retrieved by using $\mathrm{L} 1, \mathrm{~L} 2$, std L2 method

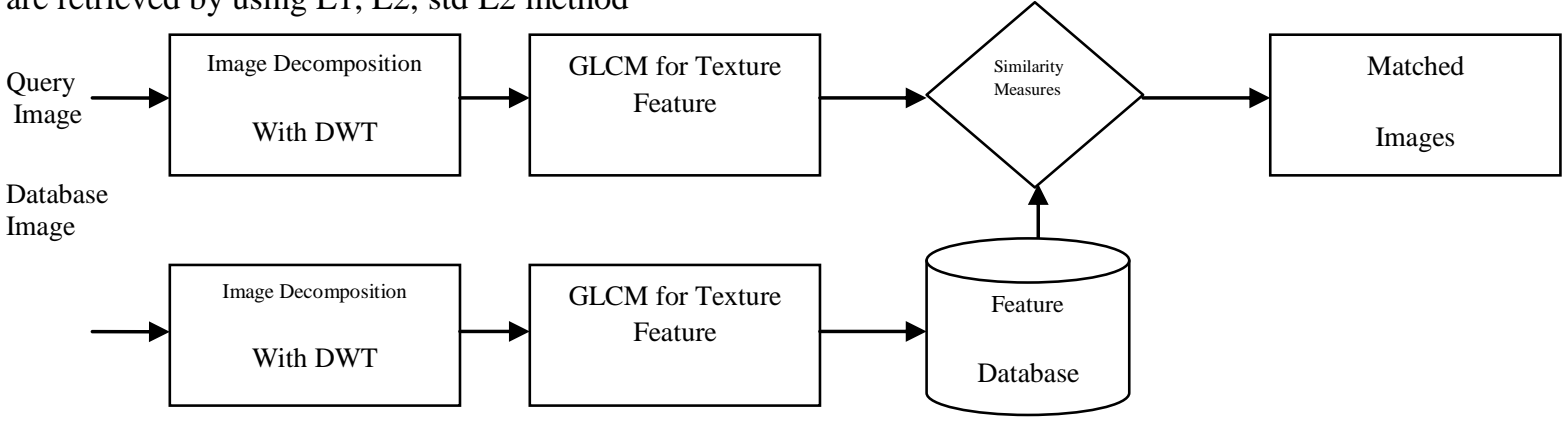

Fig. 1 Block diagram of proposed system 


\section{Discrete Wavelet Transform}

The Discrete Wavelet Transform (DWT), which is based on sub-band coding, is found to yield a fast computation of Wavelet Transform. It is easy to implement and reduces the computation time and resources required. The two-dimensional DWT of an image function $\mathrm{s}\left(\mathrm{n}_{1}, \mathrm{n}_{2}\right)$ of size $\mathrm{N}_{1} \times \mathrm{N}_{2}$ may be expressed as

$$
\begin{gathered}
W_{\varphi}\left(j_{0}, k_{1}, k_{2}\right)=\frac{1}{\sqrt{N_{1} N_{2}}} \sum_{n_{1}=0}^{N_{1}-1} \sum_{n_{2}=0}^{N_{2}-1} s\left(n_{1}, n_{2}\right) \varphi_{j_{0}, k_{1}, k_{2}}\left(n_{1}, n_{2}\right) \\
W_{\psi}^{i}\left(j_{0}, k_{1}, k_{2}\right)=\frac{1}{\sqrt{N_{1} N_{2}}} \sum_{n_{1}=0}^{N_{1}-1} \sum_{n_{2}=0}^{N_{2}-1} s\left(n_{1}, n_{2}\right) \psi_{j_{0}, k_{1}, k_{2}}^{i}\left(n_{1}, n_{2}\right)
\end{gathered}
$$

Where $\mathrm{i}=\{\mathrm{H}, \mathrm{V}, \mathrm{D}\}$ indicate the direction index of the wavelet function. As in one-dimensional case. $\mathrm{j}_{0}$ represents any starting scale, which may be treated as $\mathrm{j}_{0}=0$. Given the above two equations are two-dimensional DWT.

The speciality of DWT [6] comparing to other transforms is time and frequency characteristics which application of it wildly in many different fields.

The flow chart of the Discrete wavelet transform sub band coding on the digital image is shown in figure 2, here $\mathrm{L}$ refers to low frequency component, $\mathrm{H}$ refers to high frequency and the number 1 and 2 refer to the decomposition level of the Discrete wavelet transform. The result of the 2-D Discrete Wavelet Transform from level one to level three is shown in figure 3. The sub image LL is the low frequency component, it is the approximate sub image of the original image; the sub image HL is the component of the low frequency in horizontal direction and the high frequency in vertical direction, it manifests the horizontal edge of the original image; the sub image LH is the component of the high frequency in horizontal direction and the low frequency in vertical direction, it manifests the vertical edge of the original image; the sub image $\mathrm{HH}$ is the high frequency component, it manifests the oblique edge of the original image. It is shown that most energy of the original image is contained in the LL2 low frequency region. And the other region in the same size reflect edge feature of the image in different angles. Here we use the 2-D Discrete Haar wavelet transform for the decomposition of the images.

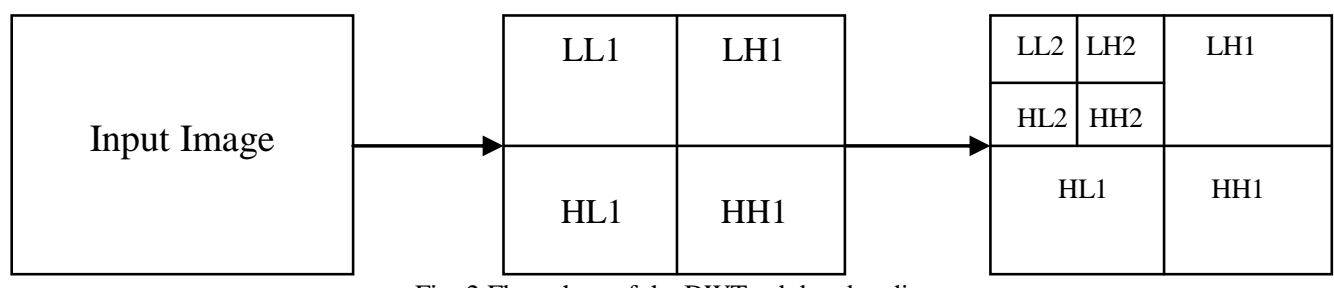

Fig. 2 Flow chart of the DWT sub band coding

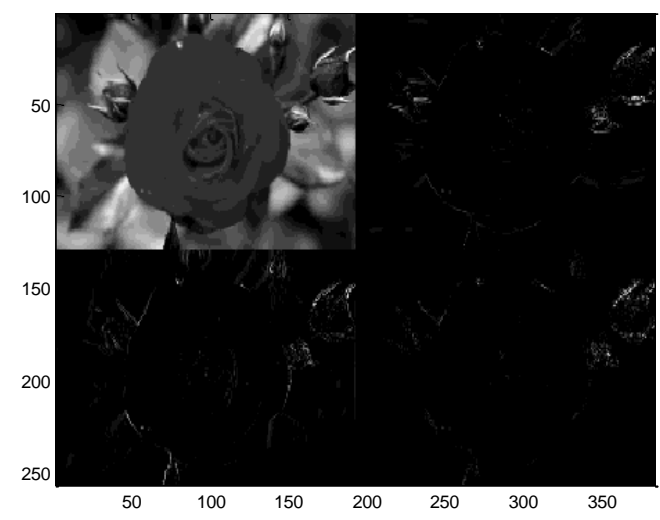

(a)

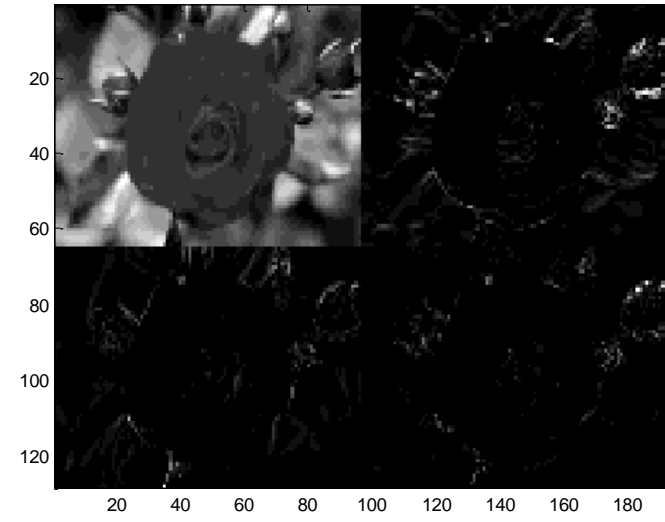

(b)

Fig. 3 (a) and (b) shows decomposition of images by level 1 and 2.

Fig. 3(a) and (b) shows the level one and level two decomposition of the image.

\section{Texture Feature Extraction}

An important feature of an image is texture. To describe the texture of the region three approaches are used in image processing these are statistical, structural and spectral. Statistical approaches specify the characterization of the textures by smooth, coarse, grainy, silky and so on. The common second order statistic is gray level co occurrence matrix. 


\subsection{Gray Level Co-Occurrence Matrix (GLCM)}

Gray Level Co-occurrence Matrix (GLCM) method is based on the conditional probability density function. GLCM introduced by Haralick. It contains the information about the positions of pixels that having similar gray level values. Co-occurrence matrix function represents the direction and distance. In the given direction and distance we can calculate the symbolic gray level pixel $\mathrm{i}, \mathrm{j}$. That can be expressed as the number of co occurrence matrix

Element $p(i, j \mid d, \theta)$.

$$
p(i, j \mid d, \theta)=\frac{p(i, j \mid d, \theta)}{\sum_{i} \sum_{j} p(i, j \mid d, \theta)}
$$

A GLCM is represented, as a matrix. In which the number of rows and columns is equal to the number of gray levels in the image. The matrix element $P(i, j \mid d, \theta)$ is the relative frequency with which two pixels, separated by distance $d$. The direction specified by the particular angle $(\theta)$.

Texture feature are computed from the statistical distribution. This can be observed combination of intensity at specified position relative to each other in the image.

Here texture feature are extracted by using gray level co-occurrences matrixes of these three properties.

\subsubsection{Homogeneity}

4.1.2 Energy

4.1.3 Correlation

\subsubsection{Homogeneity}

It returns a value that measures the closeness of the distribution of elements in the GLCM. Its range is [0 1]. Homogeneity is 1 for a diagonal GLCM.

$$
H=\sum_{i, j=0}^{N-1} \frac{P_{i, j}}{1+(i-j)^{2}}
$$

\subsubsection{Energy}

The sum of squared elements in the gray level co-occurrence matrix called Energy. Its range is [0 1].Energy is 1 for a constant image.

\subsubsection{Correlation}

$$
E=\sum_{i, j=0}^{N-1} P_{i, j}^{2}
$$

Returns a measure of how correlated a pixel is to its neighbor over the whole image. Its range is [-1 1]. Correlation is 1 or -1 for a perfectly positively or negatively correlated image. Correlation is $\mathrm{NaN}$ for a constant image.

$$
C=\sum_{i, j} \frac{(i-\mu i)(j-\mu j) p(i, j)}{\sigma_{i} \sigma_{j}}
$$

\section{Distance Measures}

Distance measures such as the Euclidean, Manhattan and Standard Euclidean distance have been used to determine the similarity of feature vectors. In this CBIR system Euclidean distance, Standard Euclidean distance and also Manhattan distance is used to commonly to compare the similarity between the images.

Distance between two images is used to find the similarities between query image and the images in the database.

\subsection{Euclidean distance}

Euclidean distance square root of the sum of the squares of the distance between corresponding values.

$$
d=\sqrt{\sum_{i=1}^{n}\left(x_{i}-y_{i}\right)^{2}}
$$

\subsection{Manhattan distance}

It computes the distance that would be travelled to get from one data point to other. The sum of the difference of their corresponding samples.

$$
d=\sum_{i=1}^{n}\left|x_{i}-y_{i}\right|
$$




\subsection{Standard Euclidean Distance}

Standardized Euclidean distance means Euclidean distance is calculated on standardized data.

Standardized value $=($ Original value - mean $) /$ Standard Deviation

$$
d=\sqrt{\sum_{i=1}^{n} \frac{1}{s_{i}^{2}}\left(x_{i}-y_{i}\right)^{2}}
$$

\section{Simulation Result And Discussion}

Simulation results are performed with MATLAB. This database consists of 1000 images each classes have 100 images for the experimental purpose we took ten images from each class totally 100 images. This experiment gives the performance comparison result of content based image retrieval for the metric L1, L2 and StdL2.Here feature extraction is done by Haar wavelet of level 2 with texture feature. Here we combine the GLCM properties of homogeneity with energy and energy with correlation to extract texture feature the comparison are done for this two combined properties with different distance classifiers.

Fig. 4 shows the Query image and retrieved images by similarity measures of the Euclidean distance for homogeneity and energy property.

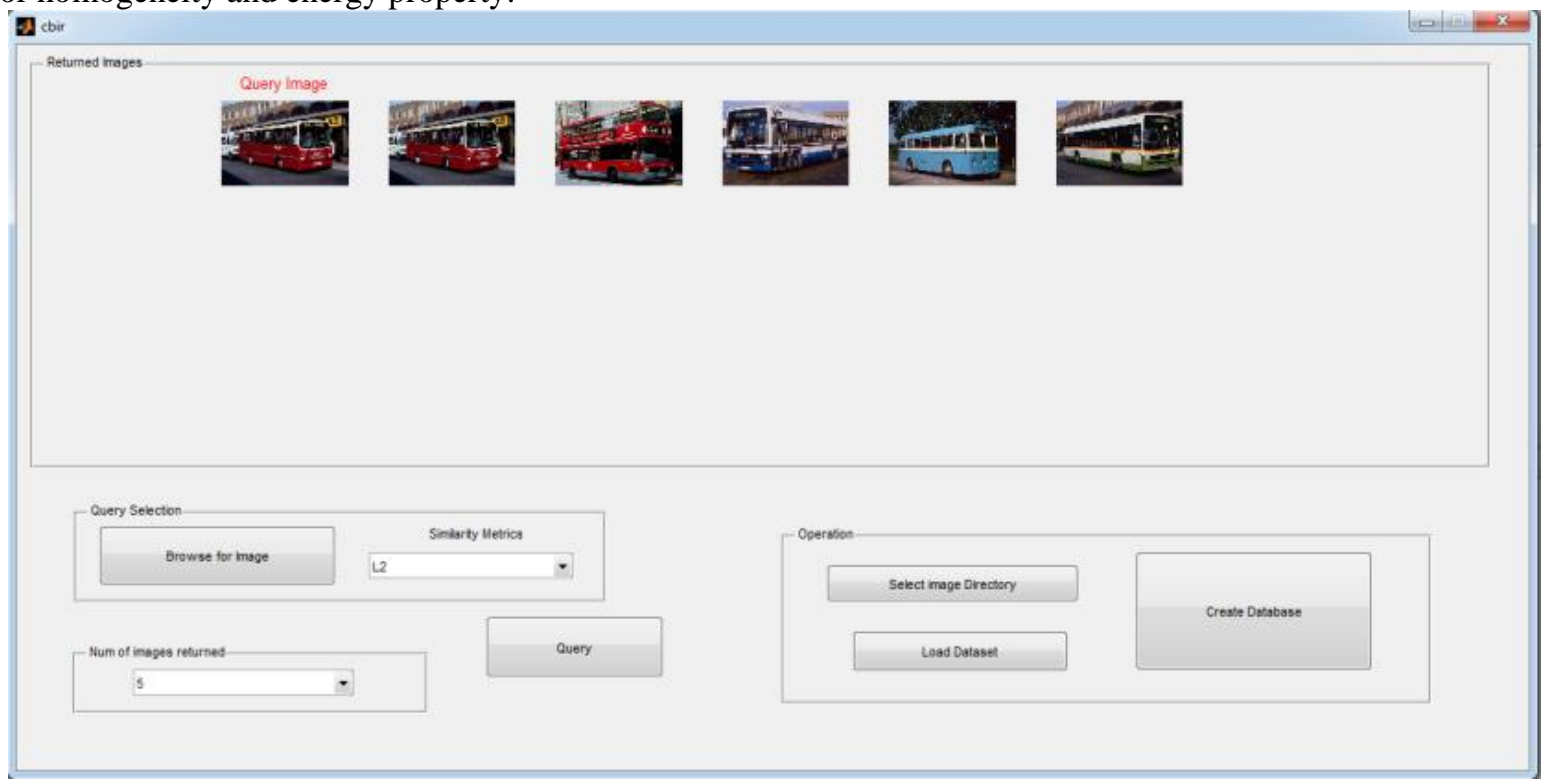

Fig. 4 Simulation Result for 2-D DWT with Texture for Euclidean Distance

Fig. 5 shows the Query image and retrieved images by similarity measures of the Manhattan distance for homogeneity and energy property.

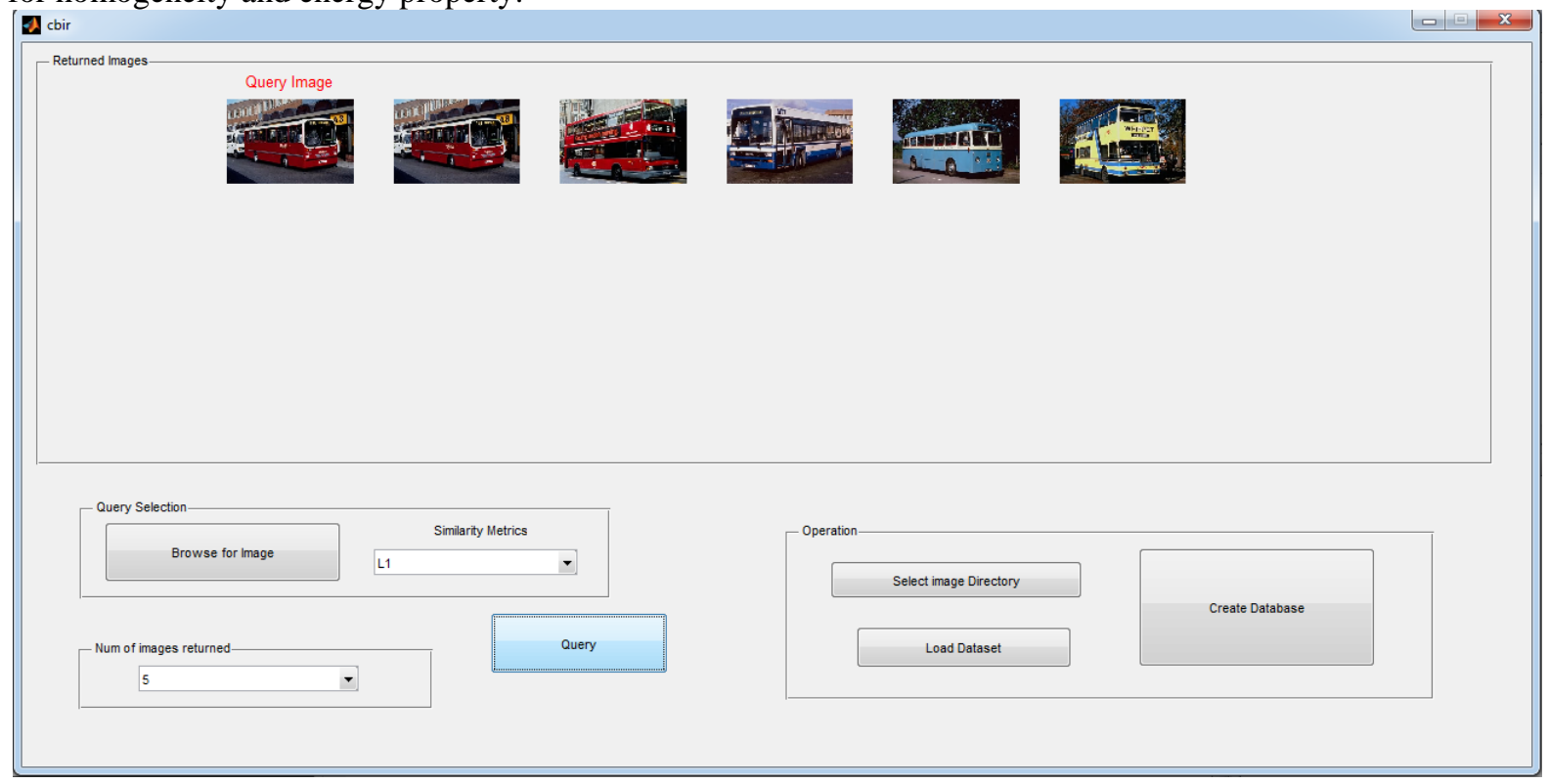

Fig.5 Simulation Result for DWT with Texture for Manhattan Distance 
Fig. 6 shows the Query image and retrieved images by similarity measures of the Standard Euclidean distance for homogeneity and energy property.

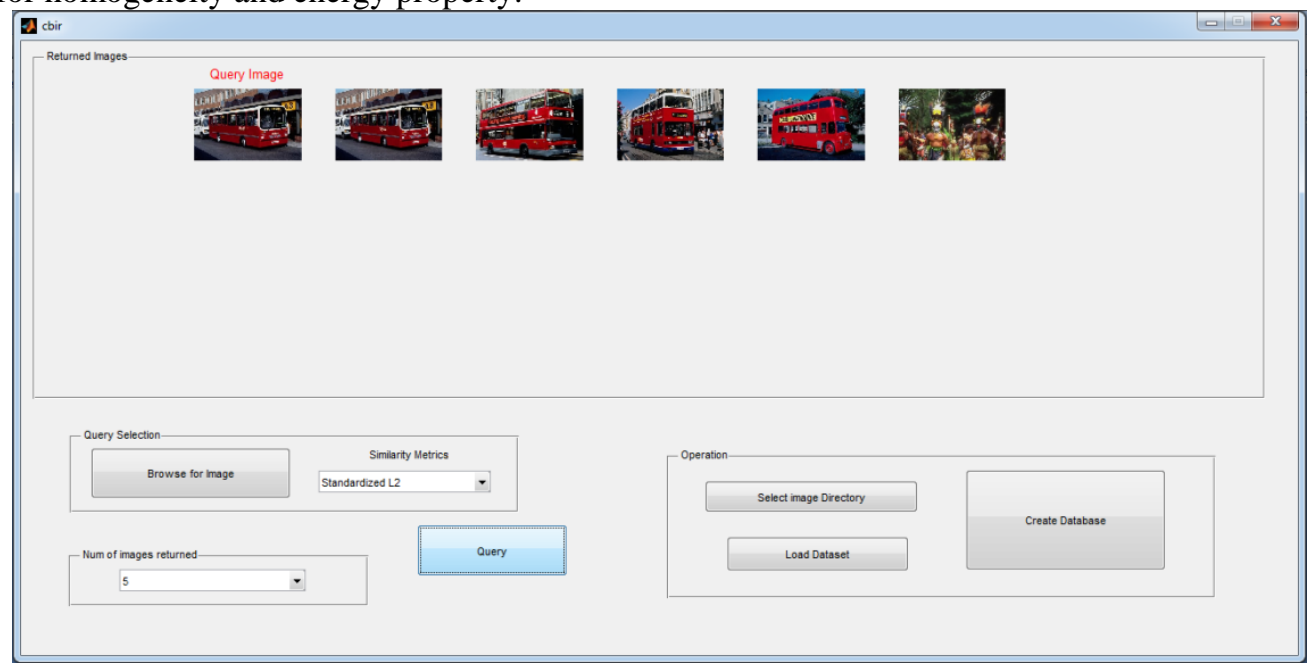

Fig.6 Simulation Result for DWT with Texture for Standard Euclidean Distance

Fig.7 shows the Query image and retrieved images by similarity measures of the Euclidean distance for correlation and energy property.

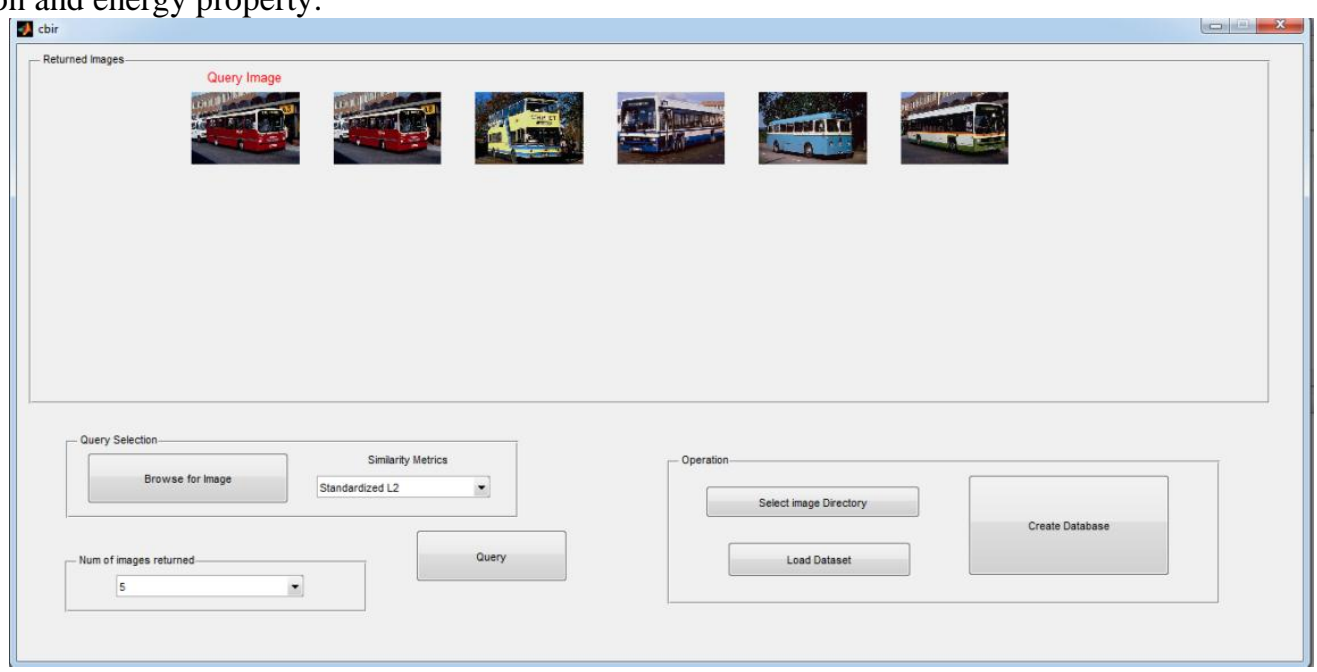

Fig. 7 Simulation Result for 2-D DWT with Texture for Euclidean Distance

Fig. 8 shows the Query image and retrieved images by similarity measures of the Manhattan distance for correlation and energy property.

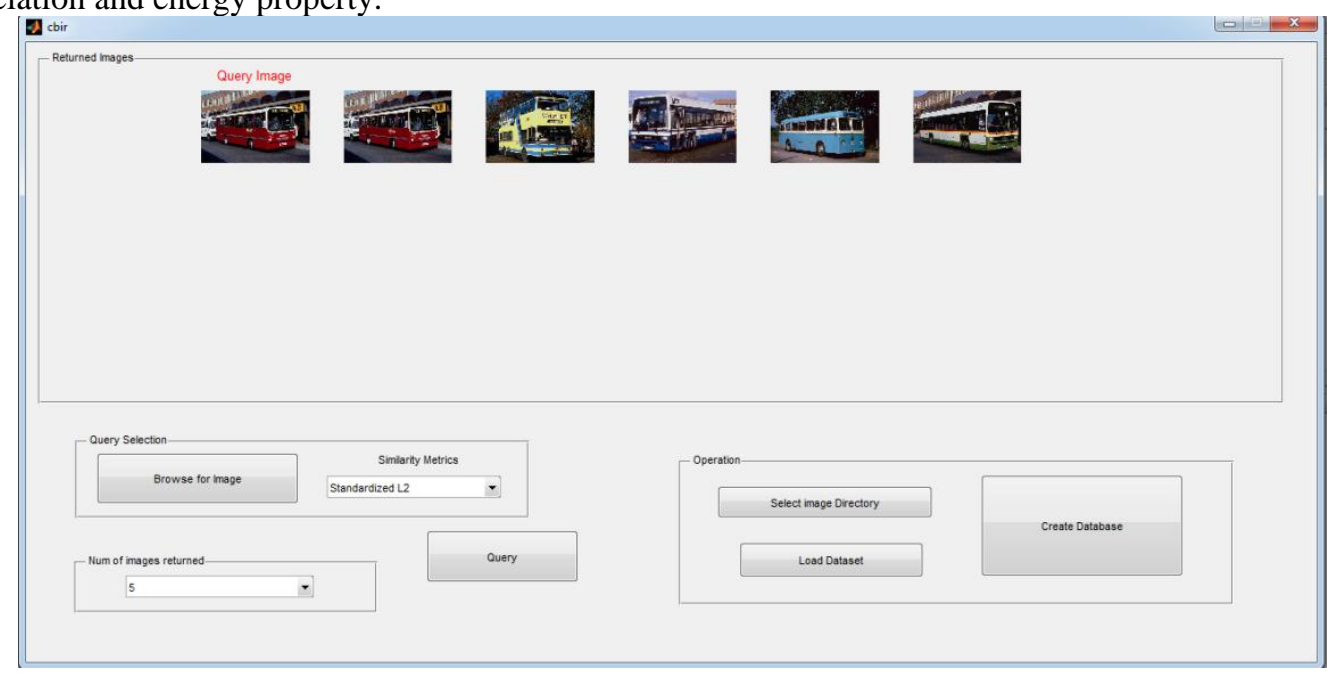

Fig. 8 Simulation Result for 2-D DWT with Texture for Manhattan Distance 
Fig. 9 shows the Query image and retrieved images by similarity measures of the Standard Euclidean distance for correlation and energy property.

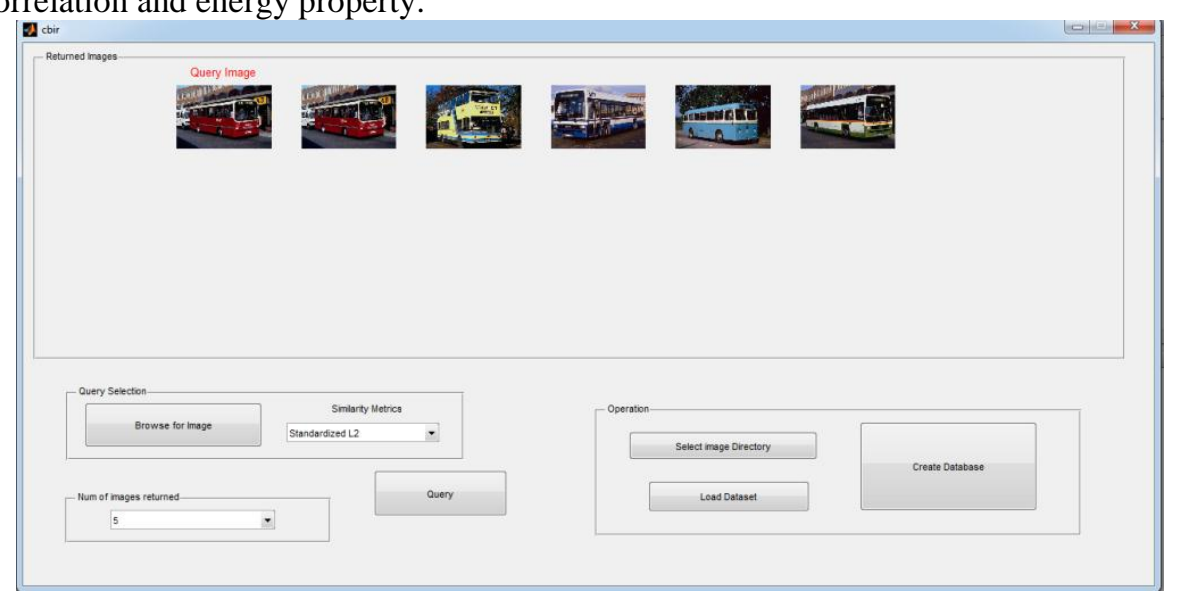

Fig. 9 Simulation Result for 2-D DWT with Texture for Standard Euclidean Distance Retrieval Accuracy is calculated by

$$
\text { Accuracy }=\frac{\text { No of Relevant Images Retrieved }}{\text { Total No of Images Retrieved }}
$$

This table shows the retrieval accuracy for each class of the CBIR system.

TABLE.1 RETRIEVAL ACCURACY

\begin{tabular}{|c|c|c|c|c|c|c|}
\hline \multirow{2}{*}{$\begin{array}{c}\text { Class } \\
\text { Name }\end{array}$} & $\begin{array}{c}\text { Manhattan } \\
\text { distance } \\
\text { L1 }\end{array}$ & $\begin{array}{c}\text { Euclidean } \\
\text { Distance } \\
\text { L2 }\end{array}$ & $\begin{array}{c}\text { Standard } \\
\text { Euclidean } \\
\text { Distance } \\
\text { Std L2 }\end{array}$ & $\begin{array}{c}\text { Manhattan } \\
\text { distance } \\
\text { L1 }\end{array}$ & $\begin{array}{c}\text { Euclidean } \\
\text { Distance } \\
\text { L2 }\end{array}$ & $\begin{array}{c}\text { Standard } \\
\text { Euclidean } \\
\text { Distance } \\
\text { Std L2 }\end{array}$ \\
\hline People & 70 & 76 & 70 & 72 & 76 & 74 \\
\hline Beach & 78 & 82 & 78 & 92 & 92 & 82 \\
\hline Building & 82 & 82 & 78 & 92 & 92 & 92 \\
\hline Buses & 94 & 96 & 92 & 94 & 94 & 84 \\
\hline Dinosaur & 96 & 96 & 94 & 100 & 100 & 100 \\
\hline Elephant & 88 & 90 & 84 & 98 & 98 & 92 \\
\hline Horses & 98 & 96 & 96 & 98 & 98 & 98 \\
\hline Roses & 88 & 86 & 84 & 76 & 80 & 86 \\
\hline Mountain & 86 & 86 & 80 & 98 & 96 & 84 \\
\hline Food & 88 & 88 & 82 & 78 & 80 & 84 \\
\hline $\begin{array}{c}\text { Average } \\
\text { Retrieval } \\
\text { Accuracy }\end{array}$ & 86.8 & 87.8 & 83.8 & 89.8 & 90.6 & 87.6 \\
\hline
\end{tabular}

\section{Conclusion}

It is observed that proposed method improves the Content Based Image Retrieval with 2-D Discrete Haar Wavelet with gray level co-occurrence matrix which is used to extract the texture feature of the images. Here we compared the result with different distance measure classifiers and also with different GLCM property. Table 1 shows performance comparison of Homogeneity with Energy and Energy with Correlation with different distance classifier in that one Euclidean distance gives best performance than Manhattan and Standard Euclidean distance for both Homogeneity with Energy and Energy with Correlation. When comparing with combined property of homogeneity with energy and energy with correlation homogeneity with energy gives the better performance.

\section{References}

[1] PiotrPorwik, AgnieszkaLisowska, "The Haar Wavelet Transform in Digital Image Processing: Its Status and Achievements".

[2] Phang Chang, PhangPiau, "Simple Procedure for the Designation of Haar Wavelet Matrices for Differential Equations", Proceeding of the International Multiconference of Engineers and Computer Scientists 2008 Vol II, March 2008.

[3] Patrick J. Van Fleet, “Discrete Haar Wavelet Transform”, PREP, Wavelet Workshop 2006.

[4] N. GnaneshwaraRao, Dr. V. Vijaya Kumara, V Venkata Krishna, "Texture Based Image Indexing and Retrieval", IJCSNS International Journal of Computer Science and Network Security, VOL.9 No.5, May 2009

[5] HosseinNezamabadi-pour and SaeidSaryazdi, "Object -Based Image Indexing and Retrieval in DCT Domain using Clustering Techniques", World Academy of Science, Engineering and Technology 32005 
[6] Hong Wang, Su Yang, Wei Liao, An Improved PCA Face Recognition Algorithm Based on the Discrete Wavelet Transform and the Support Vector Machines, International Conference on Computational Intelligence and Security Workshops 308-311,2007.

[7] http://www.fp.ucalgary.ca/mhallbey/tutorial.html

[8] Prof. SomnathSengupta, "Multi Resolution Analysis" Version 2 ECE IIT, Kharagpur

[9] J.Madhavan, K.Porkumaran, "Performance Comparison Of PCA, DWT-PCA And LWT-PCA For Face Image Retrieval", Computer Science \& Engineering: An International Journal (CSEIJ), Vol.2, No.6, December 2012 